Supporting Information for

\title{
Brush Polymers as Nanoscale Building Blocks for Hydrogel Synthesis
}

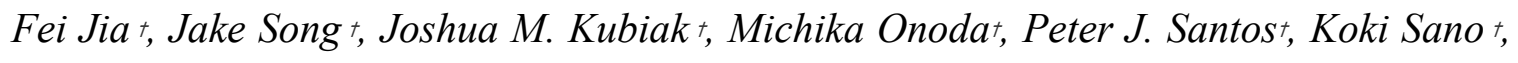
Niels Holten-Andersent, Ke Zhang $\neq$, and Robert J. Macfarlane ${ }^{+} *$

t Department of Materials Science and Engineering, Massachusetts Institute of

Technology, Cambridge, Massachusetts 02139, United States

₹ Department of Chemistry and Chemical Biology, Northeastern University, Boston, Massachusetts 02115, United States

*To whom correspondence should be addressed.

E-mail: $\underline{\text { rmacfarl@mit.edu }}$ 


\section{Materials and methods.}

Amine terminated poly (ethylene glycol) (PEG) methyl ether $\left(M_{\mathrm{n}}=3 \mathrm{kDa}\right.$, PDI $\left.<1.05\right)$ was purchased from JenKem Technology, USA. All other materials were purchased from Sigma-Aldrich Co., VWR International LLC., or Fisher Scientific Inc., and were used without further purification unless otherwise indicated. UV-Vis data were obtained on a Cary 5000 UV-Vis spectrophotometer (Varian Inc., CA, USA). DLS data were acquired on a MALVERN Zetasizer Nano-ZSP (dual-angle DLS, laser wavelength $633 \mathrm{~nm}$, light scattering angle at $12.8^{\circ}$ and $173^{\circ}$ ) and single-angle DLS (Wyatt Dyna Pro Plate Reader, laser wavelength $850 \mathrm{~nm}$, light scattering angle at 90). $N, N$-Dimethylformamide (DMF) gel permeation chromatography (GPC) was carried out on a TOSOH EcoSEC HLC-8320 GPC system (Tokyo, Japan) equipped with a TSKGel $\mathrm{GMH}_{\mathrm{HR}}-\mathrm{H}, 7.8 \times 300 \mathrm{~mm}$ column and RI/UV-Vis detectors or another DMF GPC-MALS using Agilent 1260 Infinity system with dual Agilent PL1110-6500 columns and a 0.025 M LiBr in DMF mobile phase at $60{ }^{\circ} \mathrm{C}$, in which the differential refractive index (dRI) of each compound was monitored using a Wyatt Optilab T-rEX detector. GPC is calibrated with ReadyCal-Kit PEO/PEG ( $M_{\mathrm{w}} 232$ - $941 \mathrm{kDa}$ as standards, PSS-Polymer Standards Service - USA Inc). $R_{\mathrm{g}}$ of selected high DP brush polymers were obtained with aqueous GPC-MALS (Agilent Technologies 1260 Infinity system using two Aquagel columns in water with $0.02 \%$ sodium azide as the mobile phase, GPC signals were collected using Wyatt light scattering and refractive index detectors). The dynamic mechanical properties of the hydrogels were tested on an Anton Paar rheometer with parallel plate geometry (PP-10 probe, $10 \mathrm{~mm}$ diameter, flat). Fourier transform infrared (FTIR) spectra were recorded on a Perkin-Elmer Model 2000 FT-IR spectrophotometer. For TEM analysis, samples were deposited on carbon-coated copper grids (Ted Pella Inc., CA, USA) for 5 min before the excess liquid was carefully wicked away by filter paper. The grids were then stained by pipetting $10 \mu \mathrm{L}$ of $1 \%$ uranyl acetate directly onto the grid. The stain was allowed to stay for 3 min before being wicked away. All TEM samples were imaged on an FEI Tecnai Multipurpose Digital TEM electron microscope utilizing an accelerating voltage of $120 \mathrm{kV}$. Matrix-assisted laser desorption ionization-time of flight mass spectrometry (MALDI-ToF MS) measurements were carried out on a Bruker Microflex LT mass spectrometer (Bruker Daltonics Inc., MA, USA) using a sinapinic acid matrix. 


\subsection{Syntheses of norbornenyl N-hydroxysuccinimidyl ester (N-NHS) and norbornenyl PEG monomers (N-PEG, N-PEG-Boc).}
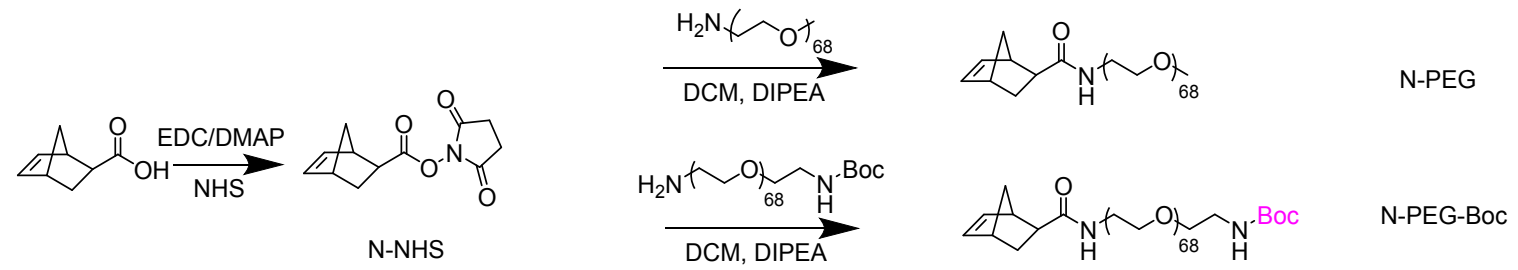

Norbornenyl hydroxysuccinimidyl ester (N-NHS) and norbornenyl PEG (N-PEG) were synthesized according to a previously reported method. ${ }^{1}$ Briefly, N-NHS was synthesized by the esterification reaction between norbornenyl carboxylic acid ( $2.0 \mathrm{~g}, 1$ equiv.) and $\mathrm{N}$ hydroxysuccinimide (NHS, 2.3 g, 1.4 equiv.) using $N$-(3-Dimethylaminopropyl)-N'ethylcarbodiimide hydrochloride ( $\mathrm{EDC} \mathrm{HCl}, 3.9 \mathrm{~g}, 1.4$ equiv.) as a coupling agent in dichloromethane $(20 \mathrm{~mL})$. N-NHS was purified by silica column $(2: 1$ hexanes/ethyl acetate as eluent) to afford a white solid product with $80 \%$ yield.

${ }^{1} \mathrm{H}-\mathrm{NMR}\left(400 \mathrm{MHz}, \mathrm{CDCl}_{3}\right): \delta$ 6.20-6.12 (m, 2H), 3.27 (s, 1H), 3.00 (s, 1H), $2.84(\mathrm{~s}, 4 \mathrm{H})$, $2.50(\mathrm{~m}, 1 \mathrm{H}), 2.05(\mathrm{~m}, 1 \mathrm{H}), 1.55-1.50(\mathrm{~m}, 1 \mathrm{H}), 1.48-1.40(\mathrm{~m}, 2 \mathrm{H}) ;{ }^{13} \mathrm{C}-\mathrm{NMR}(300 \mathrm{MHz}$, $\left.\mathrm{CDCl}_{3}\right): \delta 171.9,169.5,138.8,135.5,47.4,46.6,42.0,40.5,31.3,25.8$.

N-PEG and N-PEG-Boc macromonomers were obtained using the reported method ${ }^{2}$. In general, amine-terminated poly(ethylene glycol) (PEG) methyl ether (1.0 g, 1.0 equiv.) and N-NHS (94 mg, 1.2 equiv.) were dissolved in anhydrous dichloromethane in a round bottom flask, to which $N, N$-diisopropyl ethyl amine (DIPEA, $116 \mu \mathrm{L}, 2.0$ equiv.) was added via a pipette. The reaction mixture was allowed to stir overnight at room temperature. Next, the reaction mixture was concentrated and precipitated in $100 \mathrm{~mL}$ icecold diethyl ether $3 \times$. The resulting white precipitant was dried in vacuo $(1.0 \mathrm{~g}$ obtained, yield 93\%). 


\subsection{General method for the synthesis of brush polymers.}

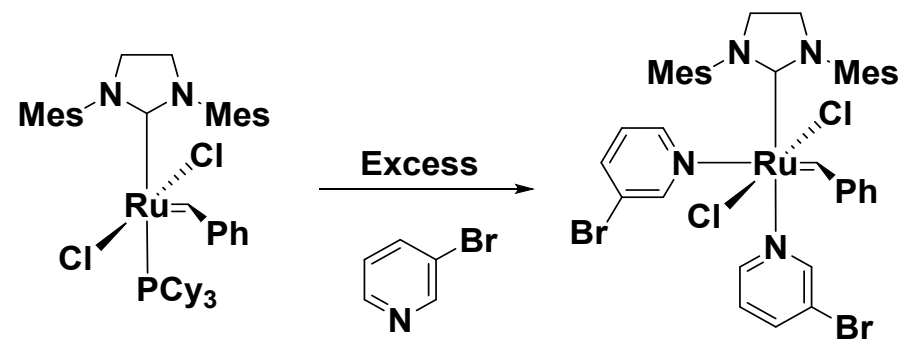

Modified $2^{\text {nd }}$ generation Grubbs' catalyst was synthesized based on a published protocol ${ }^{3}$. In brief, $2^{\text {nd }}$ generation Grubbs's catalyst $(500 \mathrm{mg})$ and excess 3-bromopyridine $(1.0 \mathrm{~mL})$ were vortexed in a $15 \mathrm{~mL}$ centrifuge tube. The reaction mixture was then precipitated in anhydrous pentane $(10 \mathrm{~mL}) 3 \times$. The resulting green powder was dried in vacuo and stored under $-20^{\circ} \mathrm{C}$. A typical polymerization process for random copolymer brushes is as follows (example given for the $\mathrm{DP}_{100-15 \%}$ random copolymer). All apparatuses and chemical compounds were first transferred to a glove box. Then, mixtures of monomers $(85 \mathrm{mg} \mathrm{N}$ PEG and $15 \mathrm{mg}$ N-PEG-Boc) were dissolved in anhydrous DCM $(0.6 \mathrm{~mL})$ in a $5 \mathrm{~mL}$ Schlenk flask, and the catalyst $(0.295 \mathrm{mg})$ was dissolved in DCM $(0.2 \mathrm{~mL})$ in a $1.5 \mathrm{~mL}$ microcentrifuge tube; this catalyst solution was then injected into the flask via a microsyringe. The reaction mixture was cooled to $\sim-10{ }^{\circ} \mathrm{C}$ by using an ice-salt bath and stirred overnight. At the end of the reaction, excess ethyl vinyl ether (EVE, $0.2 \mathrm{~mL}$ ) was added to the mixture to ensure polymerization termination and the reaction was stirred for an additional $2 \mathrm{~h}$. The mixture was then concentrated and precipitated into ethyl ether $3 \times$, and the precipitant was dried in vacuo.

For sequence-controlled block copolymers ("side-by-side, SBS" and "end-to-end, ETE" brush polymers), the same protocol noted above was followed with slight modification, as the monomers for each block were sequentially injected into the reaction mixture. "Sideby-side" polymers consisted of a middle block (target DP of 190 or 170) consisting of 5\% or $15 \%$ reactive side chains that was flanked by non-modified PEG brushes. "End-to-end" polymers had the inverse design with a central block of PEG brushes without reactive side chains (DP of 190 or 170) flanked on either end by azide/DBCO modified brushes. In a typical polymerization of ETE brushes with 5\% reactive side chains, N-PEG-Boc $(5 \mathrm{mg})$ was first dissolved in DCM $(0.1 \mathrm{~mL})$ in a Schlenk flask and catalyst $(0.295 \mathrm{mg})$ dissolved 
in $0.1 \mathrm{~mL}$ DCM was added via pipette. The N-PEG-Boc block was allowed to polymerize for $1 \mathrm{~h}$ at $-10^{\circ} \mathrm{C}$ by using an ice-salt bath. Unfunctionalized PEG monomer N-PEG (190 $\mathrm{mg}$, dissolved in 1.2 mL DCM) was subsequently injected into the flask and allowed to polymerize for another $6 \mathrm{~h}$ before the injection of the macromonomers comprising the final block (N-PEG-Boc, dissolved in $0.2 \mathrm{~mL}$ DCM). The polymerization was left to stir overnight, followed by an injection of $0.2 \mathrm{~mL}$ EVE to ensure that all polymerizations had stopped. The polymerization kinetics of the "end-to-end" brush synthesis (as monitored by DMF GPC) are shown in Figure 6b.

Typical monomer conversion during ROMP using this modified version of Grubb's $2^{\text {nd }}$ generation catalyst polymerization was observed to be nearly quantitative (Figure S2), as has been reported in the previous literature ${ }^{4,5}$. The full consumption of all macromonomers was verified using GPC measurements performed before purification (via precipitation in ether) of the polymerization product; this data is shown in Figure $1 \mathrm{~b}$.

For high DP polymers (DP $=100,150$, and 200), minimal amounts of unpolymerized macromonomer were observed (less than $1.5 \mathrm{wt} \%$ based on the GPC RI curve integration, the image of GPC-MALS is attached as Figure S3a). The trace amount of unreacted macromonomer was removed in the subsequent extensive dialysis ( 2 or 3 days of dialysis against Nanopure ${ }^{\mathrm{TM}}$ water using a dialysis membrane with molecular weight cut-off of 12$14 \mathrm{kDa})$.

\subsection{General method for the post-modification of brush polymers and 4-arm PEGs.}

For brush polymers, the Boc protecting groups were removed before post-modification by stirring the polymers in an 80/20 volume mixture of DCM/trifluoroacetic acid (TFA) for $15 \mathrm{~h}$. The resulting acidic solution was neutralized using excess sodium bicarbonate buffer. Then, the solution containing the deprotected brush polymer was collected and dialyzed against Nanopure ${ }^{\mathrm{TM}}$ water to desalt. The final solution was lyophilized to yield white solids (yield: 95\%). Complete removal of Boc protecting groups was confirmed by the disappearance of the characteristic peak in ${ }^{1} \mathrm{H}$ NMR spectra at $\sim 1.5 \mathrm{ppm}$ (Figure S2).

Post-modification of PEG-containing polymers was achieved via an amidation reaction between amine-terminated polymers and NHS ester azide or dibenzocyclooctyne (DBCO) 
(Scheme S1b). In a typical reaction for the conjugation of brush polymer and DBCO NHSester, DBCO NHS-ester (5 mg, 4.6 equiv.) and amine-terminated polymer (50 mg, 2.5 $\mu \mathrm{mol})$ were dissolved in $0.1 \mathrm{M}$ carbonate/bicarbonate solution and stirred overnight at 4 ${ }^{\circ} \mathrm{C}$. The reaction mixture was passed through a NAP-25 column (G.E. Health) to remove salts and small filtrates. The modified polymer was then lyophilized and stored at $-20{ }^{\circ} \mathrm{C}$. The typical yield for the modification was $90 \%$, and the successful synthesis of the azidemodified polymers was confirmed via FTIR (Figure S6a) and the synthesis of the DBCO modified polymers was confirmed with UV-Vis spectroscopy (Figure S6b) and ${ }^{1} \mathrm{H}$ NMR (Figure S7).

\subsection{General method for $\boldsymbol{R}_{\mathrm{h}}$ and $\boldsymbol{R}_{\mathrm{g}}$ measurements.}

$R_{\mathrm{h}}$ for each polymer was measured from two DLS instruments (dual-angle Zetasizer; single angle-Wyatt Dyna Pro Plate Reader). DLS measurements were carried out at a polymer weight concentration of $1 \mathrm{mg} / \mathrm{mL}$ in Nanopure ${ }^{\mathrm{TM}}$ water at room temperature. All samples were passed through a syringe filter (Nylon, $0.45 \mu \mathrm{m}$, VWR, US) to remove impurities prior to measurement. Cumulant fit results (provided by DLS software) were used for later calculations of $C^{*} . R_{\mathrm{h}}$ values, determined as a function of $\mathrm{q}^{2}$ for different polymers, are provided in Figure S8. $q$ is calculated based on the equation: $q=4 \pi n / \lambda * \sin (\theta / 2)$, where $n$ is the refractive index of water (1.33), $\lambda$ is the laser wavelength $(633 \mathrm{~nm}$ and $850 \mathrm{~nm}$ respectively), and $\theta$ is the scattering angle $\left(12.8^{\circ}, 90^{\circ}\right.$, and $\left.173^{\circ}\right)$. Raw data measured from different angles are plotted in Figure S8.

$R_{\mathrm{g}}$ values for the brush polymer gel precursors with backbone DP of 75, 100, 150, and 200 with $15 \%$ DBCO modifications were determined via aqueous GPC-MALS. $R_{\mathrm{g}}$ values for brush polymers with lower DPs $(4,25$, and 50) are smaller than $10 \mathrm{~nm}$ and therefore not appropriate for MALS analysis (typical MALS detection range $R_{\mathrm{g}}$ is $\sim 10-500 \mathrm{~nm}$ ). The $R_{\mathrm{g}} / R_{\mathrm{h}}$ results for each brush polymer precursor are listed in Table $\mathrm{S} 2$.

\subsection{General method for hydrogel formation.}

Gelation for both brush polymer and 4-arm PEG hydrogels was achieved by mixing the DBCO and azide modified counterparts. DBCO and azide-modified polymers were first dissolved in water in separate microcentrifuge tubes and sonicated for at least $20 \mathrm{~min}$ to 
make sure the polymers were well dissolved. Then, the azide-modified polymer solution was injected into the DBCO modified polymer solution via pipette and thoroughly mixed. The reaction mixture was then sealed to prevent water evaporation and incubated in an oven at $45^{\circ} \mathrm{C}$ for $1 \mathrm{~h}$, then cooled down to room temperature or overnight for further characterizations.

\subsection{General procedure for rheology measurements.}

A Peltier hood with a water cell was used for all experiments to control the temperature during measurements and to prevent sample dehydration. Dynamic oscillatory strain amplitude sweep measurements were conducted at a frequency of $10 \mathrm{rad} / \mathrm{s}$ to determine the linear viscoelastic region (LVR, Figure S16); storage modulus $\left(G^{\prime}\right)$ and loss modulus $\left(G^{\prime \prime}\right)$ were measured as a function of strain amplitude ranging from 1 to $100 \%$ on a logarithmic scale. Frequency sweep measurements were conducted at a 5\% strain amplitude and angular frequency ranging from 10 to $0.1 \mathrm{rad} / \mathrm{s}$ on a logarithmic scale while monitoring $G^{\prime}$ and $G^{\prime \prime}$. The determination of which samples had formed fully networked gels was determined as a function of the value of Tan $\delta\left(G^{\prime \prime} / G^{\prime}\right)$ at $10 \mathrm{rad} / \mathrm{s}, 25^{\circ} \mathrm{C}$.

Gelation tests were performed by measuring the $G^{\prime}$ and $G^{\prime \prime}$ immediately after placing the azide and DBCO polymer solution mixtures on the sample stage. Then, $G^{\prime}$ and $G^{\prime \prime}$ were monitored over time by oscillatory measurements $(\tau=10 \mathrm{~Pa}, \omega=10 \mathrm{rad} / \mathrm{s})$ at $45^{\circ} \mathrm{C}$. For these tests, the polymer samples were sealed by a layer of mineral oil to prevent dehydration during the testing period.

\subsection{General procedure for the hydrogel swelling measurements.}

In a typical experiment, $200 \mu \mathrm{L}$ of $10 \mathrm{wt} \%$ polymer hydrogel was prepared with phosphate-buffered saline (PBS) in a microcentrifuge tube. Once the gel had formed, $1 \mathrm{~mL}$ PBS buffer was put on top of the hydrogel, and the centrifuge tube was subsequently placed on a thermomixer stirred at $300 \mathrm{rpm}$ at $37^{\circ} \mathrm{C}$. PBS buffer was removed at predetermined time points $(1,2,4,8,12,24,48$, and $72 \mathrm{~h})$, and the weights of the hydrogel and microcentrifuge tube were measured. The wet mass change of a piece of hydrogel was used to evaluate the gel's degree of swelling. 
So

Scheme S1. (a) Synthetic scheme and structures for synthesizing PEG macromonomers. Synthesis and post-modification of brush polymer (b) and 4-arm PEGs (c).

a

PEG
macromonomer

b

Brush polymer synthesis

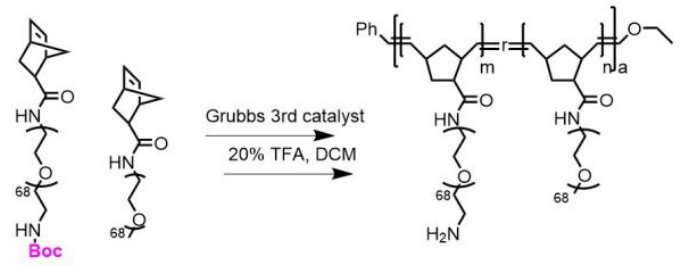

C

4-arm star polymer synthesis
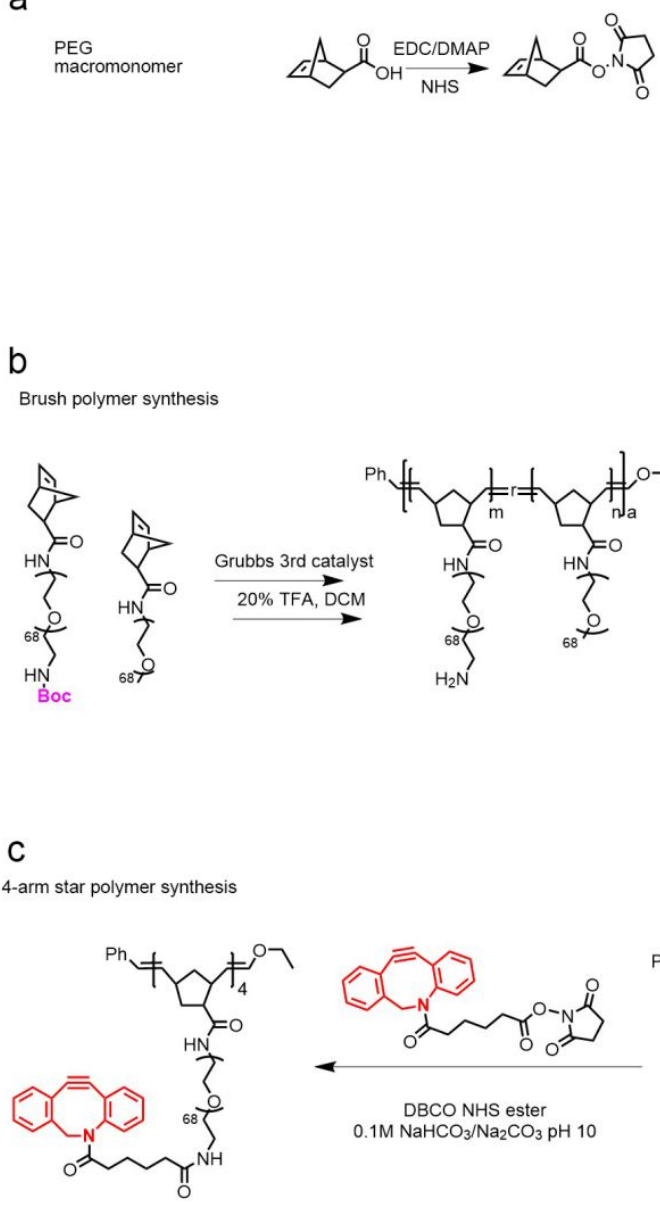
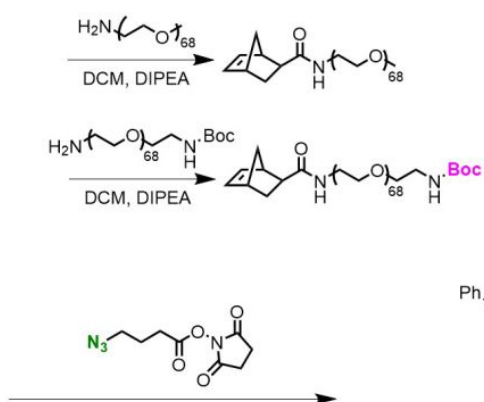

Azide $\mathrm{NHS}$ ester
$0.1 \mathrm{M} \mathrm{NaHCO} / \mathrm{Na}_{2} \mathrm{CO}_{3} \mathrm{pH} 10$

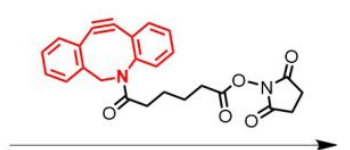

DBCO NHS ester $0.1 \mathrm{M} \mathrm{NaHCO} / \mathrm{Na}_{2} \mathrm{CO}_{3} \mathrm{pH} 10$
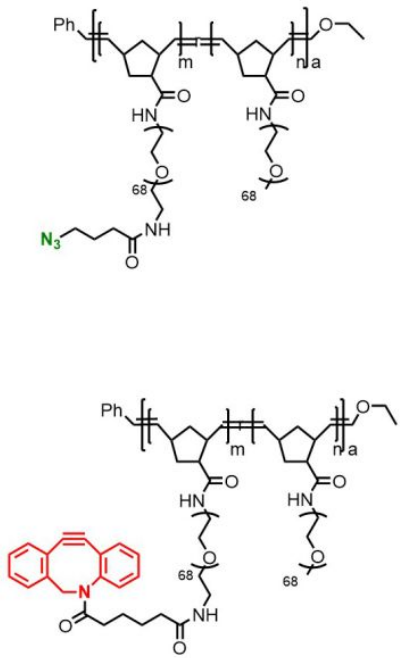
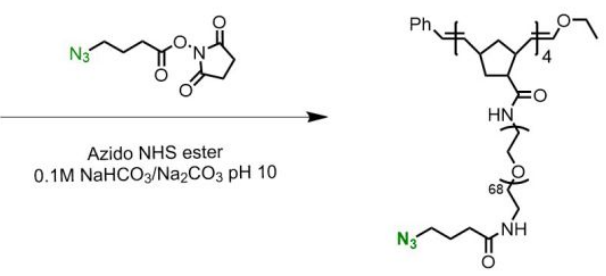
Table S1. GPC Analyses for the 4-arm PEG and brush polymers Used.

\begin{tabular}{|c|c|c|c|c|c|c|c|}
\hline Polymer & $M_{\mathrm{n}} \mathrm{kDa}$ & $M_{\mathrm{w}} \mathrm{kDa}$ & $\boldsymbol{\oplus}$ & Polymer & $M_{\mathrm{n}} \mathrm{kDa}$ & $M_{\mathrm{w}} \mathrm{kDa}$ & $\boldsymbol{D}$ \\
\hline $\mathrm{DP}_{50-5 \%}$ & 183.5 & 227.5 & 1.24 & $\mathrm{DP}_{150-10 \%}$ & 388.0 & 554.8 & 1.43 \\
\hline DP $_{50-10 \%}$ & 195.2 & 245.9 & 1.26 & DP $_{150-15 \%}$ & 418.9 & 640.9 & 1.53 \\
\hline DP $_{50-15 \%}$ & 197.6 & 254.9 & 1.29 & $\mathrm{DP}_{200-5 \%}$ & 557.0 & 794.9 & 1.55 \\
\hline DP $_{75-5 \%}$ & 249.2 & 301.6 & 1.21 & $\mathrm{DP}_{200-10 \%}$ & 566.9 & 907.0 & 1.60 \\
\hline DP $_{75-10 \%}$ & 258.5 & 341.2 & 1.32 & $\mathrm{DP}_{200-15 \%}$ & 609.7 & 1048.6 & 1.72 \\
\hline $\mathrm{DP}_{75-15 \%}$ & 250.7 & 343.6 & 1.37 & SBS $_{200-5 \%}$ & 602.4 & 987.9 & 1.64 \\
\hline DP $_{100-5 \%}$ & 317.8 & 419.5 & 1.32 & SBS $_{200-15 \%}$ & 671.2 & 1141.1 & 1.70 \\
\hline DP $_{100-10 \%}$ & 316.5 & 446.3 & 1.41 & ETE $_{200-5 \%}$ & 662.4 & 1053.2 & 1.59 \\
\hline$D_{100-15 \%}$ & 332.6 & 482.3 & 1.45 & ETE $_{200-15 \%}$ & 651.8 & 1049.4 & 1.61 \\
\hline $\mathrm{DP}_{150-5 \%}$ & 376.7 & 516.1 & 1.37 & 4-arm PEG & 12.0 & 13.9 & 1.16 \\
\hline$D_{P_{25-f}=4}$ & 134.3 & 167.9 & 1.25 & $\mathrm{DP}_{25-100 \%}$ & 135.8 & 170.2 & 1.25 \\
\hline $\mathrm{DP}_{50-100 \%}$ & 199.5 & 309.2 & 1.55 & $\mathrm{DP}_{75-100 \%}$ & 260.2 & 426.7 & 1.64 \\
\hline $\mathrm{DP}_{100-100 \%}$ & 350.2 & 609.3 & 1.74 & & & & \\
\hline
\end{tabular}

Table S2. $R_{\mathrm{g}} / R_{\mathrm{h}}$ ratio of four brush polymers (15\% DBCO modified brush polymers)

\begin{tabular}{|c|c|c|c|}
\hline $\mathbf{D P}$ & $\boldsymbol{R}_{\mathbf{g}}(\mathbf{n m})$ & $\boldsymbol{R}_{\mathrm{h}}(\mathbf{n m})$ & $\boldsymbol{R}_{\mathrm{g}} / \boldsymbol{R}_{\mathrm{h}}$ \\
\hline $\mathbf{7 5}$ & 11.9 & 20.2 & 0.59 \\
$\mathbf{1 0 0}$ & 15.9 & 22.0 & 0.72 \\
$\mathbf{1 5 0}$ & 19.5 & 27.7 & 0.70 \\
$\mathbf{2 0 0}$ & 25.0 & 34.6 & 0.72 \\
\hline
\end{tabular}


Table S3. Estimated $C^{*}$ for different polymers based on the $D_{\mathrm{h}}$ measure by DLS. $R_{\mathrm{g}}$ was estimated with the spherical structure approximation that $R_{\mathrm{g}} / R_{\mathrm{h}}=0.774$. For large brush polymers ( $\mathrm{DP}=75,100,150,200), R_{\mathrm{g}}$ was directly measured with aqueous GPC equipped with multi-angle light scattering.

\begin{tabular}{|c|c|c|}
\hline Polymer & $\boldsymbol{R}_{\mathbf{g}}(\mathbf{n m})$ & $\boldsymbol{C}^{*} \mathbf{( w t \% )}$ \\
\hline 4-arm & $\sim 3.4$ & $\sim 12.8$ \\
\hline $\mathbf{D P}_{\mathbf{2 5}}$ & $\sim 6.2$ & $\sim 8.2$ \\
\hline $\mathbf{D P}_{\mathbf{5 0}}$ & $\sim 8.2$ & $\sim 7.1$ \\
\hline $\mathbf{D P}_{\mathbf{7 5}}$ & $\sim 11.9$ & $\sim 5.6$ \\
\hline $\mathbf{D P}_{\mathbf{1 0 0}}$ & $\sim 15.9$ & $\sim 3.2$ \\
\hline $\mathbf{D P}_{\mathbf{1 5 0}}$ & $\sim 19.5$ & $\sim 2.2$ \\
\hline $\mathbf{D P}_{\mathbf{2 0 0}}$ & $\sim 25.0$ & $\sim 1.5$ \\
\hline
\end{tabular}




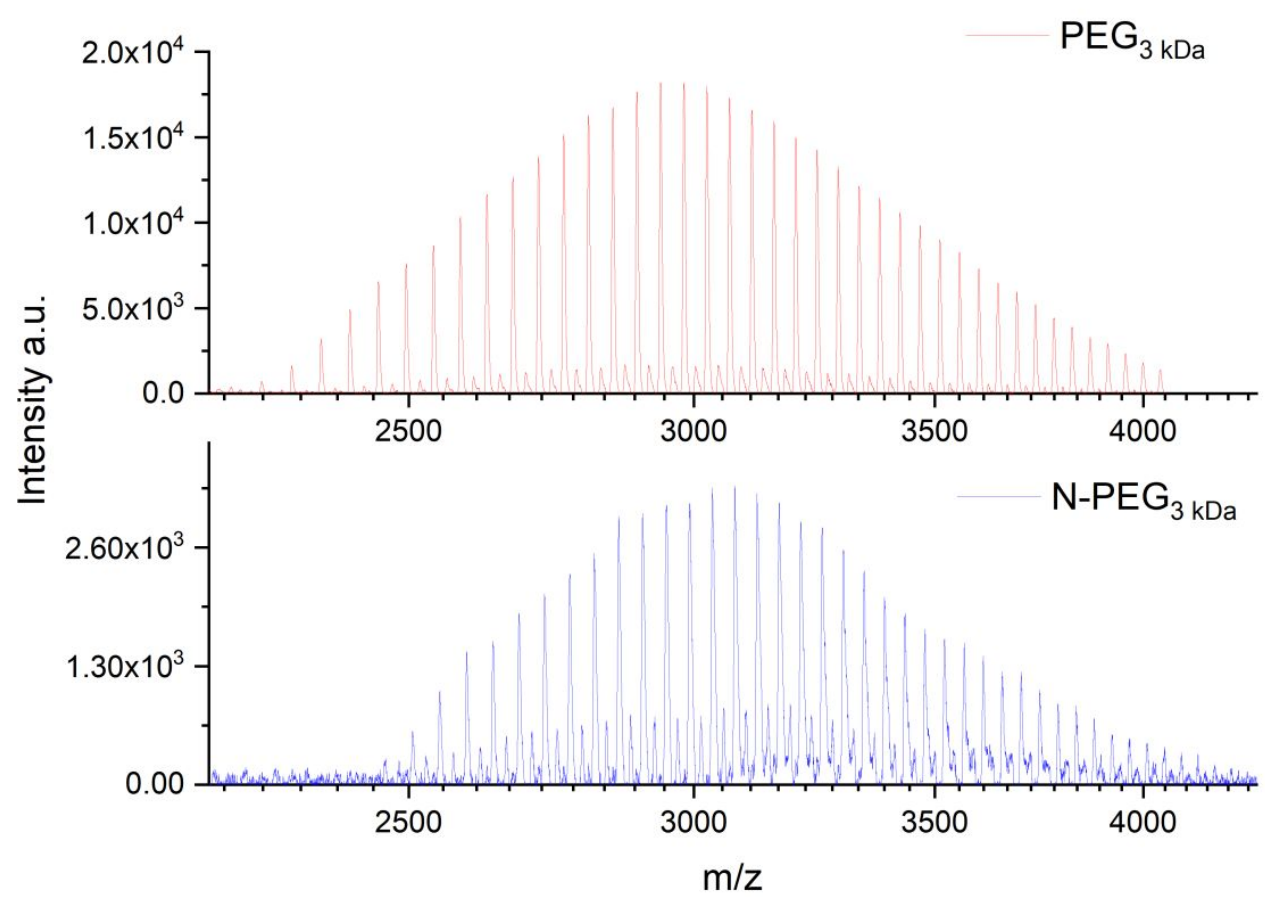

Figure S1. MALDI-MS of amine terminated PEG (3 kDa) and N-PEG $(\sim 3.2 \mathrm{kDa})$ macromonomer using sinapinic acid matrix.

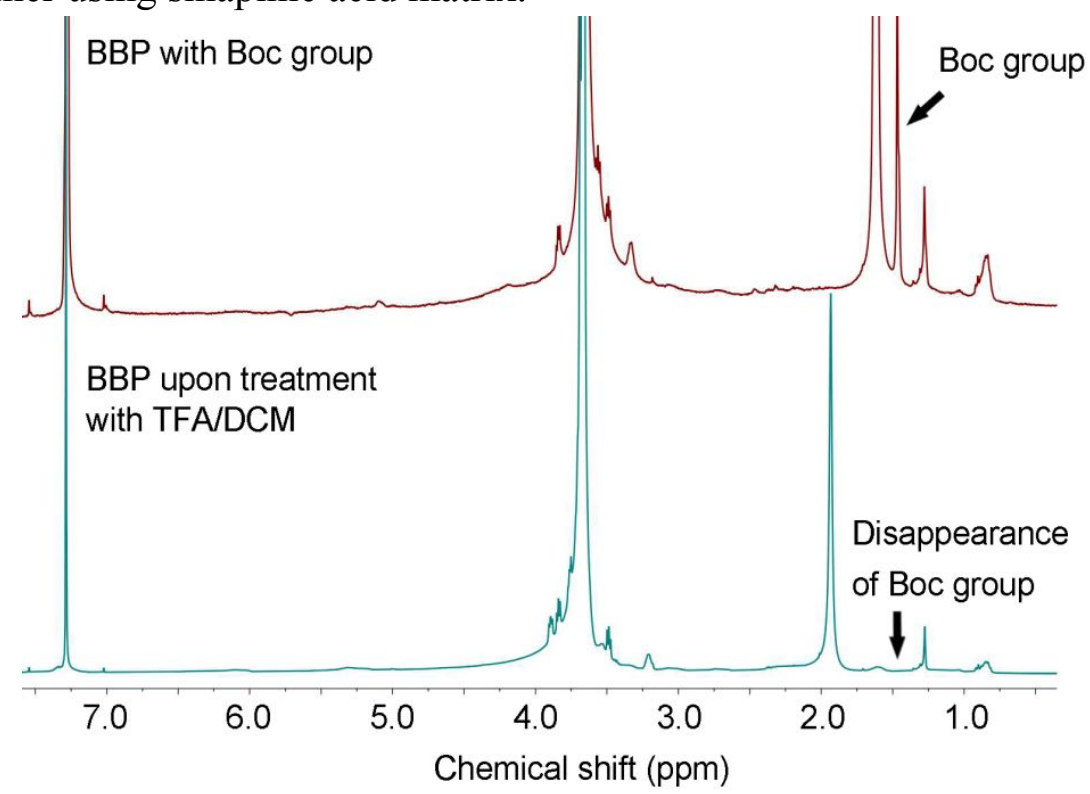

Figure S2. ${ }^{1} \mathrm{H}$ NMR spectrums of polymer (4-arm PEG polymerized with norborneneterminated $3 \mathrm{kDa}$ PEG) with Boc protecting group and polymer upon Boc protecting group removal by TFA/DCM in d-chloroform expanded in the region of the chain-end protons, showing the complete removal of Boc group. 

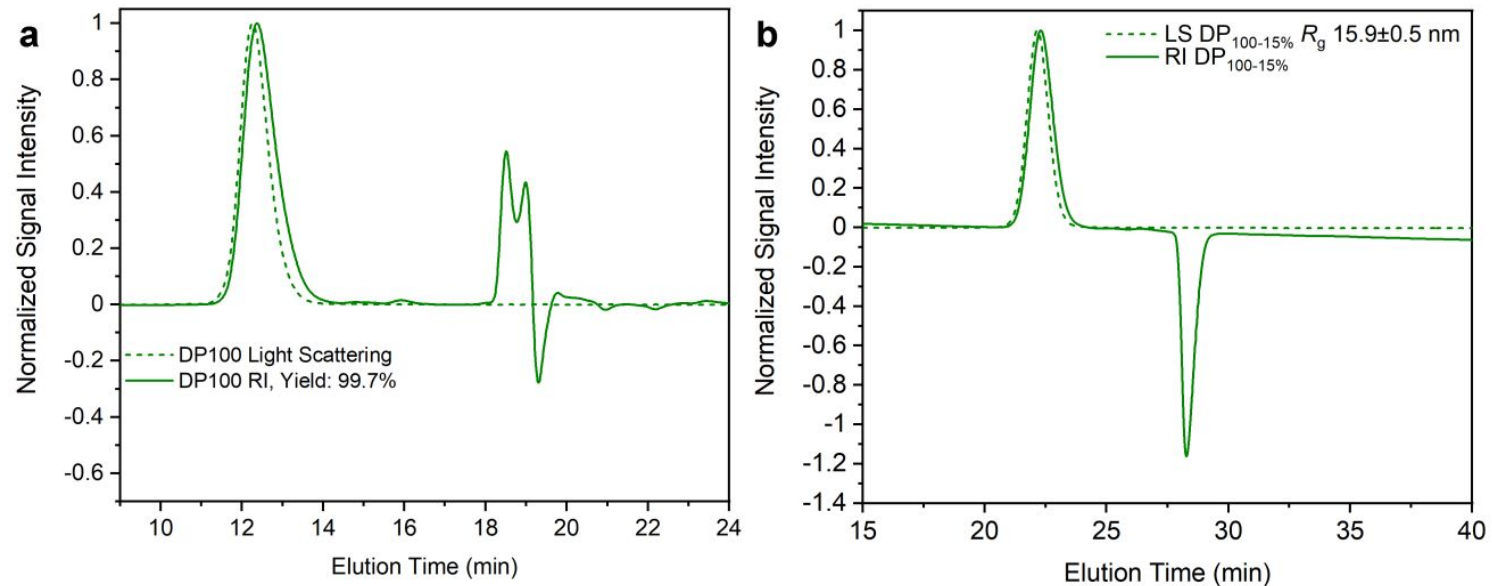

Figure S3. (a) DMF GPC-MALS traces (dot line: light scattering signal; solid line: RI detector signal) of brush polymer with DP 100 (15\% Boc-PEGs), yield calculation is based on the integration of RI signal. (b) Aqueous GPC-MALS traces (dot line: light scattering signal; solid line: RI detector signal) of brush polymer with DP 100 (15\% DBCO groups), $R_{\mathrm{g}}$ results are based on the fitting of multi-angle light scattering detector.

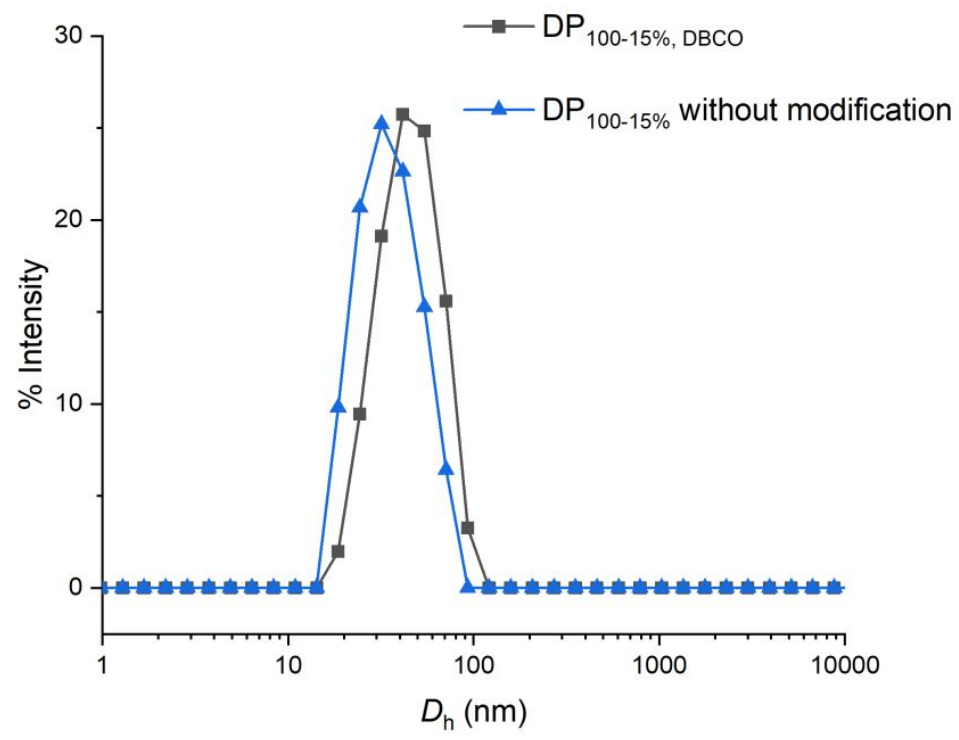

Figure S4. Size distributions of $\mathrm{DP}_{100}$ brush polymer before and after the $\mathrm{DBCO}$ modification. 


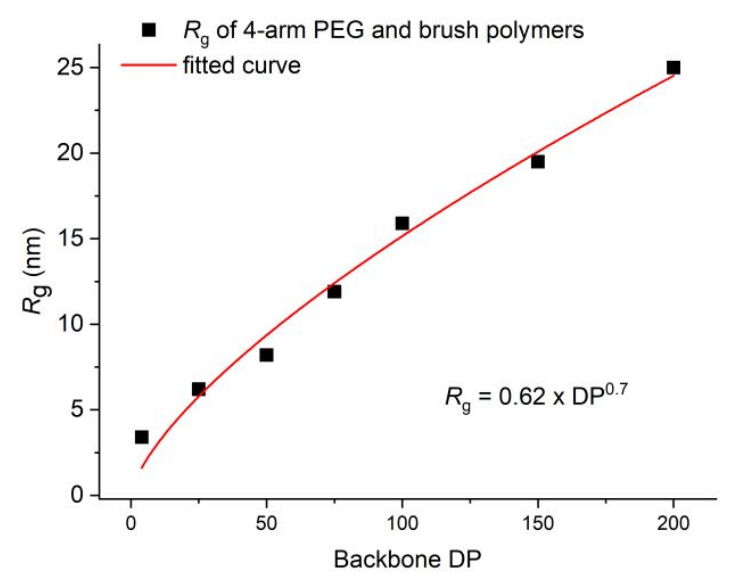

Figure S5. Change of $R_{\mathrm{g}}$ as a function of the backbone DP of polymers.
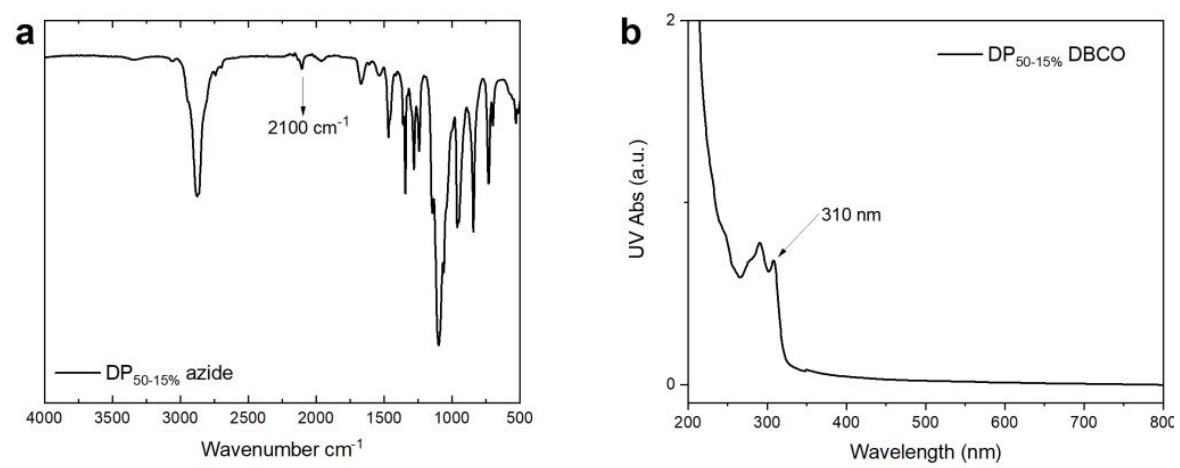

Figure S6. (a) FTIR spectrum of $\mathrm{DP}_{50 \text {-azide. }}$ Arrow points to azide stretching vibration. (b). $\mathrm{UV}$-vis spectrum of $\mathrm{DP}_{50-15 \%-\mathrm{DBCO}}$. Arrow points to DBCO absorption.

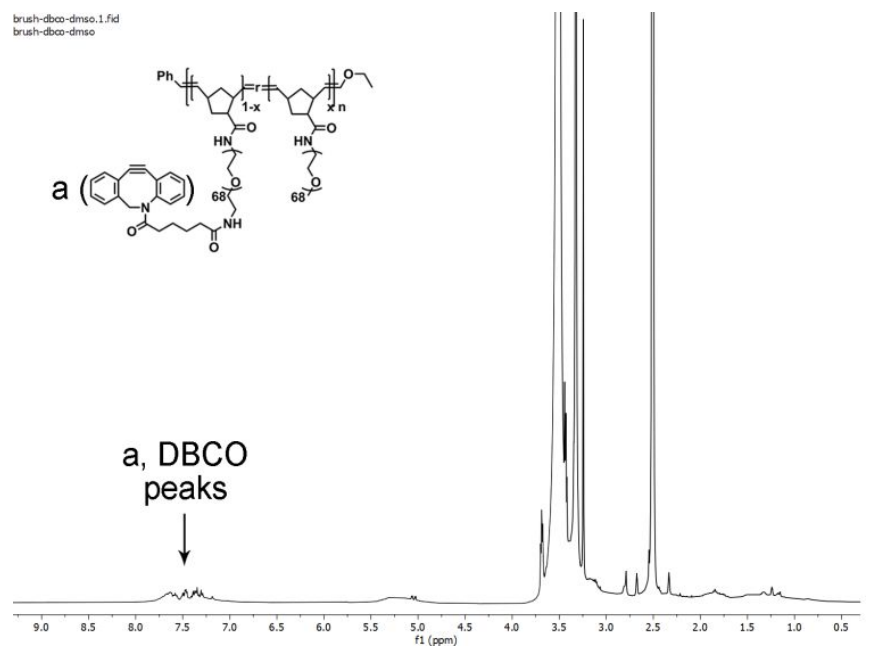

Figure S7. ${ }^{1} \mathrm{H}$ NMR spectrum of DBCO functionalized brush polymer, $\mathrm{DP}_{25-f=4}$. 


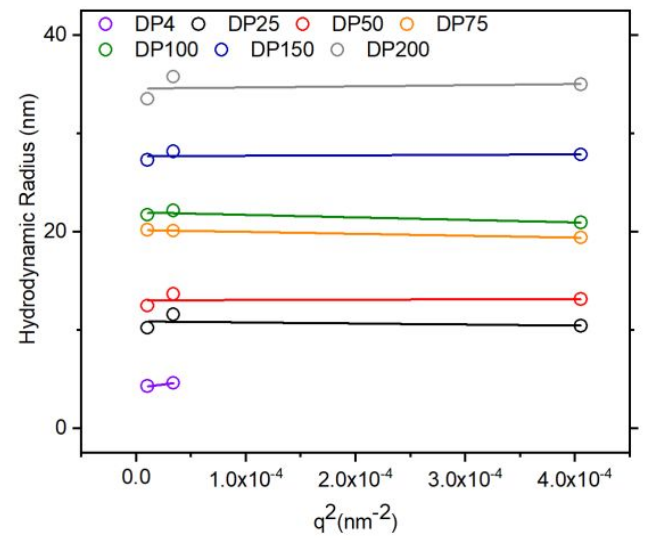

Figure S8. Hydrodynamic radius $\left(R_{\mathrm{h}}\right)$ as a function of $\mathrm{q}^{2} \cdot R_{\mathrm{h}}$ were obtained from 2 DLSs.
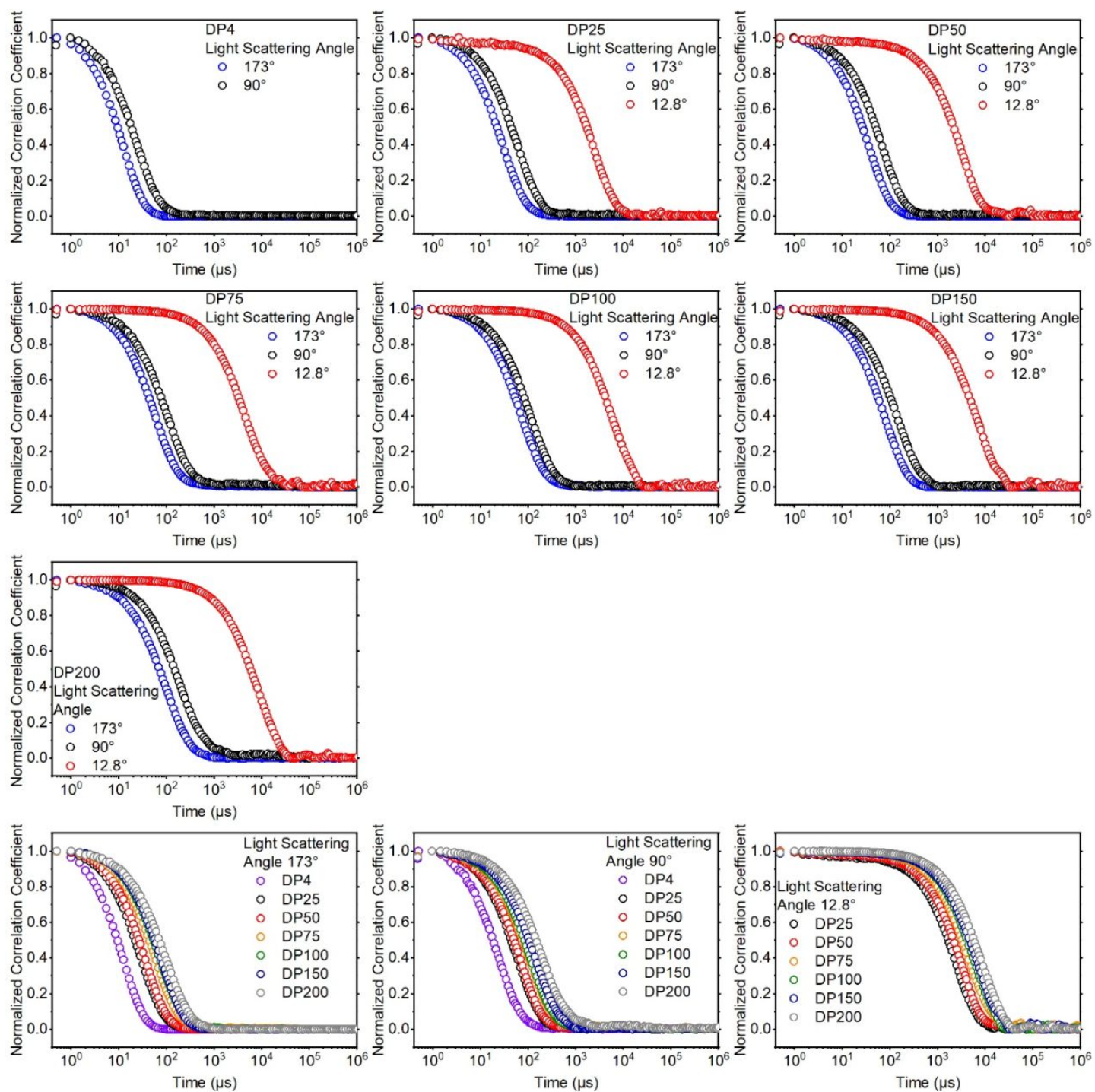
Figure S9. Correlation function for polymers with three different scattering angles $\left(12.8^{\circ}\right.$, $\left.90^{\circ}, 173^{\circ}\right)$.
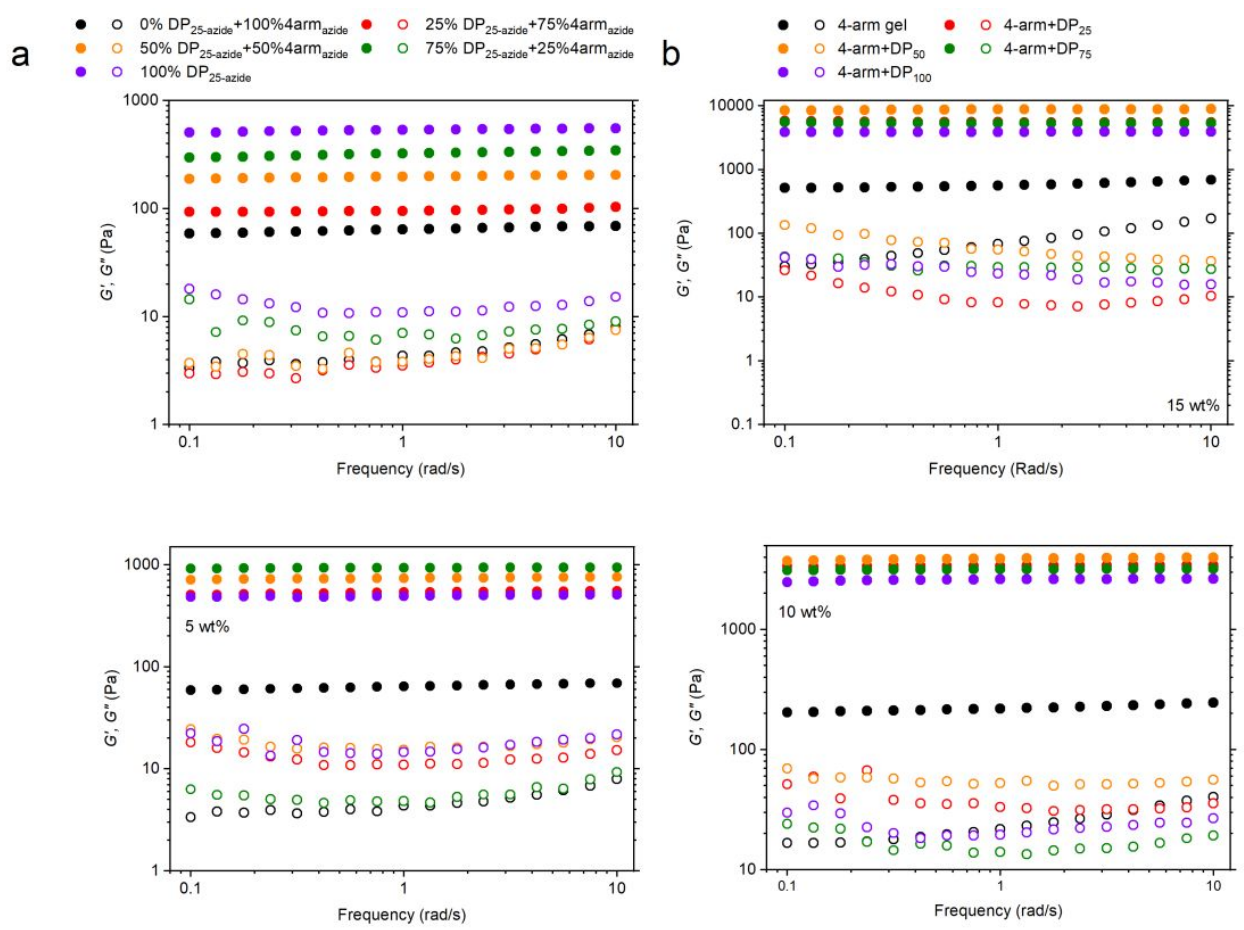

Figure S10. Frequency dependent $\left(\epsilon=5 \%, 25^{\circ} \mathrm{C}\right)$ rheology measurements of PEG hydrogels. $G^{\prime}$, solid dots; $G^{\prime \prime}$, empty dots. (a) Substituting azide modified 4-arm PEG with $\mathrm{DP}_{25 \text {-azide }}$ in the 4-arm PEG gels. (b) Gels crosslinked by brush polymers and 4-arm PEG at different polymer concentrations (5 wt $\%, 10 \mathrm{wt} \%$, and $15 \mathrm{wt} \%)$.

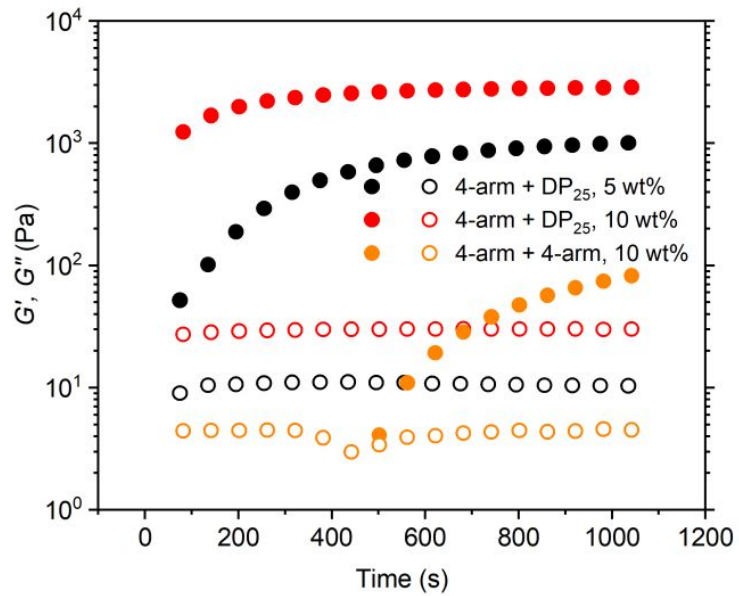

Figure S11. Gelation kinetics of gels composed of DBCO modified 4-arm PEG and $\mathrm{DP}_{25}$ - 
azide at $5 \mathrm{wt} \%$ and $10 \mathrm{wt} \%$, respectively. $\mathrm{DP}_{25}$ can form solid gels instantly as evidenced by the value of $G^{\prime}$ is larger than $G^{\prime \prime}$ at the beginning of gelation kinetics measurement.
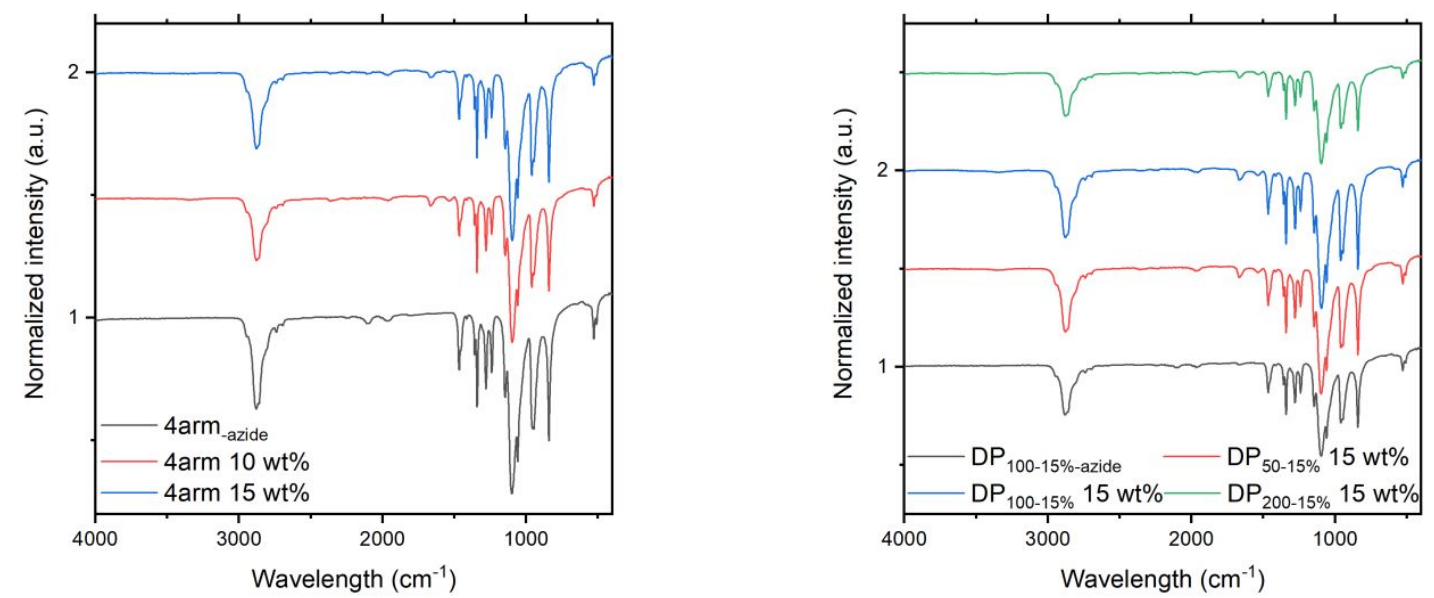

Figure S12. FTIR spectra of the azide modified polymer and lyophilized polymer network after gelation.

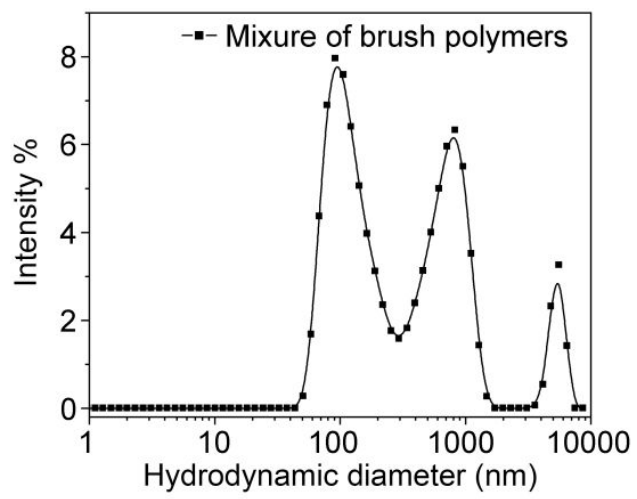

Figure S13. DLS analysis of polymer mixture of $\mathrm{DP}_{100-5 \%}$, DLS experiments were carried out at a scattering angle of $173^{\circ}$ at room temperature. 


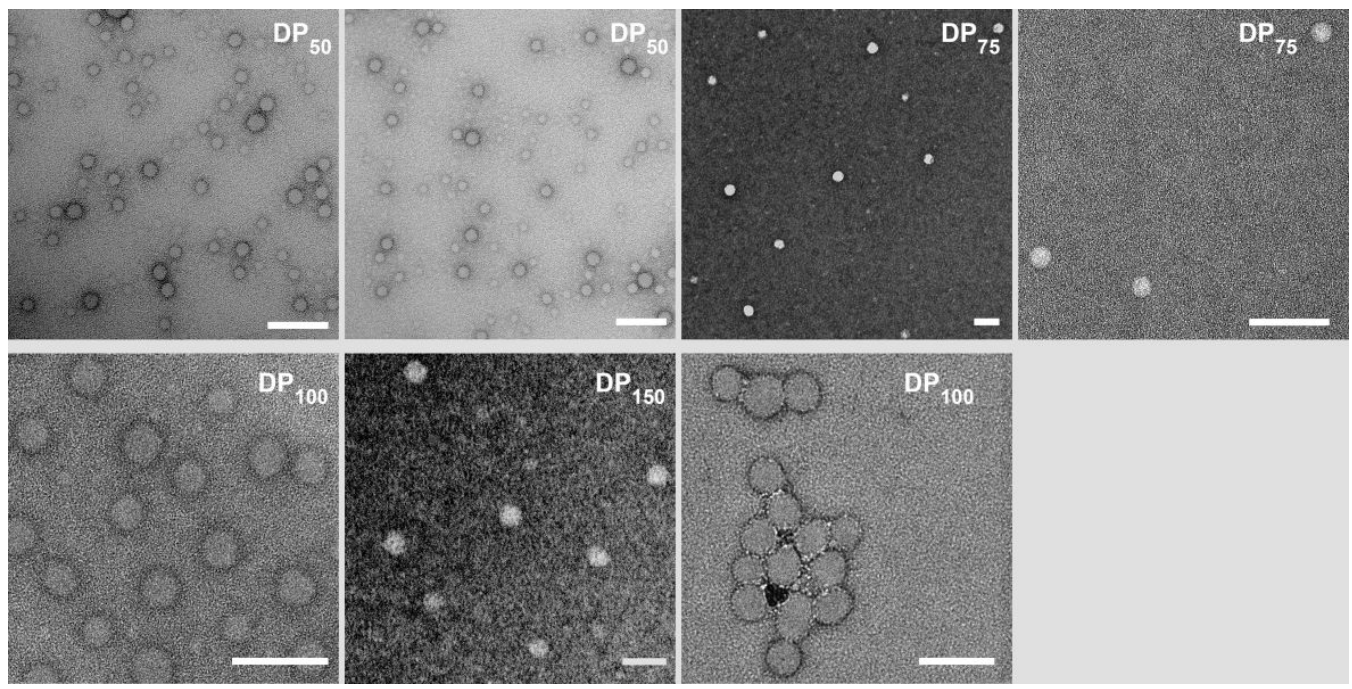

Figure S14. TEM images of individual brush polymers and assembled structures following addition of their counterparts $\left(\mathrm{DP}_{100-15 \%}\right)$, both prepared from dilute polymer solutions (1 $\mathrm{wt} \%)$ and subsequently stained with uranium acetate (1\%). Scale bar: $100 \mathrm{~nm}$.
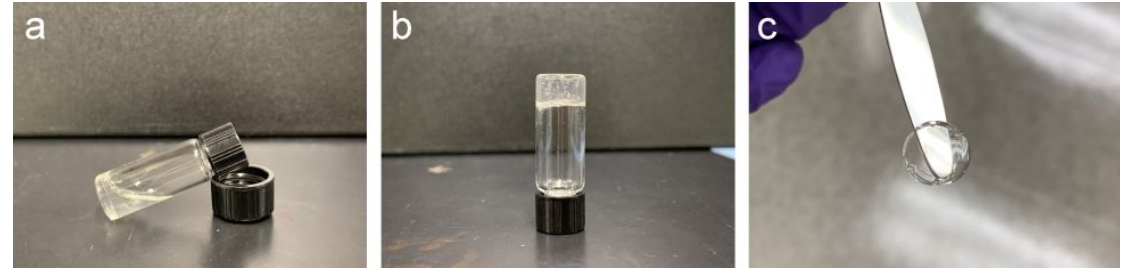

Figure S15. Brush polymer mixture $\mathrm{DP}_{100-15 \%}$ before (a) and after (b and c) gelation.

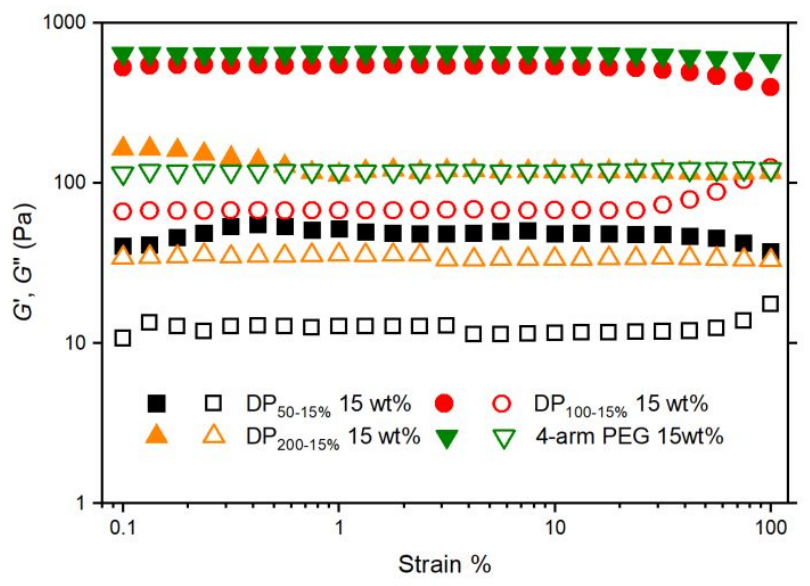

Figure S16. Strain dependent $\left(\omega=10 \mathrm{rad} / \mathrm{s}, 25^{\circ} \mathrm{C}, 15 \mathrm{wt} \%\right)$ rheology measurements of selected brush polymer hydrogels and 4-arm PEG hydrogel. $G^{\prime}$, solid dots; $G^{\prime \prime}$, empty dots. 

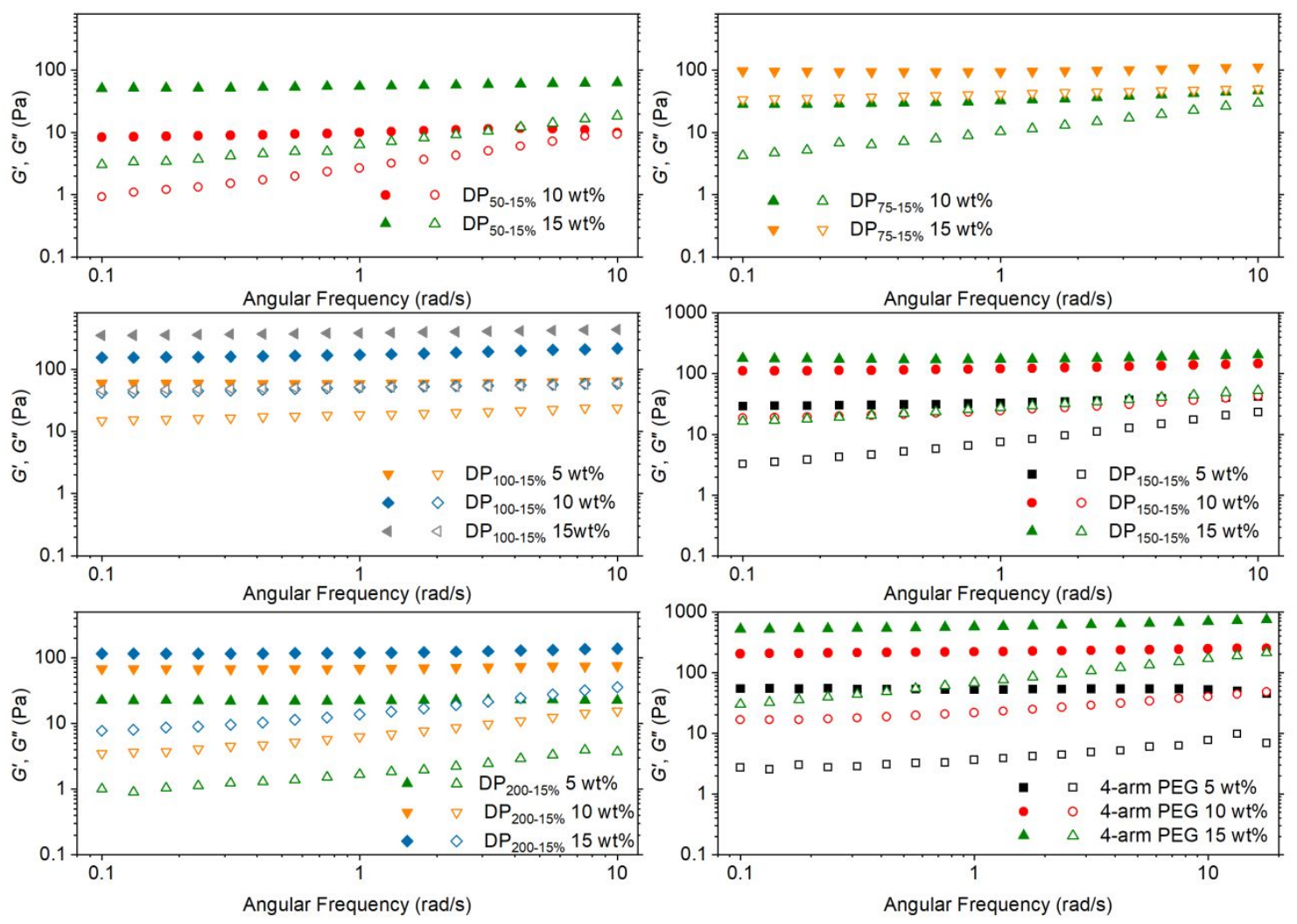

Figure S17. Frequency dependent $\left(\epsilon=5 \%, 25^{\circ} \mathrm{C}, 5,10,15\right.$ wt. $\left.\%\right)$ rheology measurements of PEG hydrogels. $G^{\prime}$, solid dots; $G^{\prime \prime}$, empty dots.
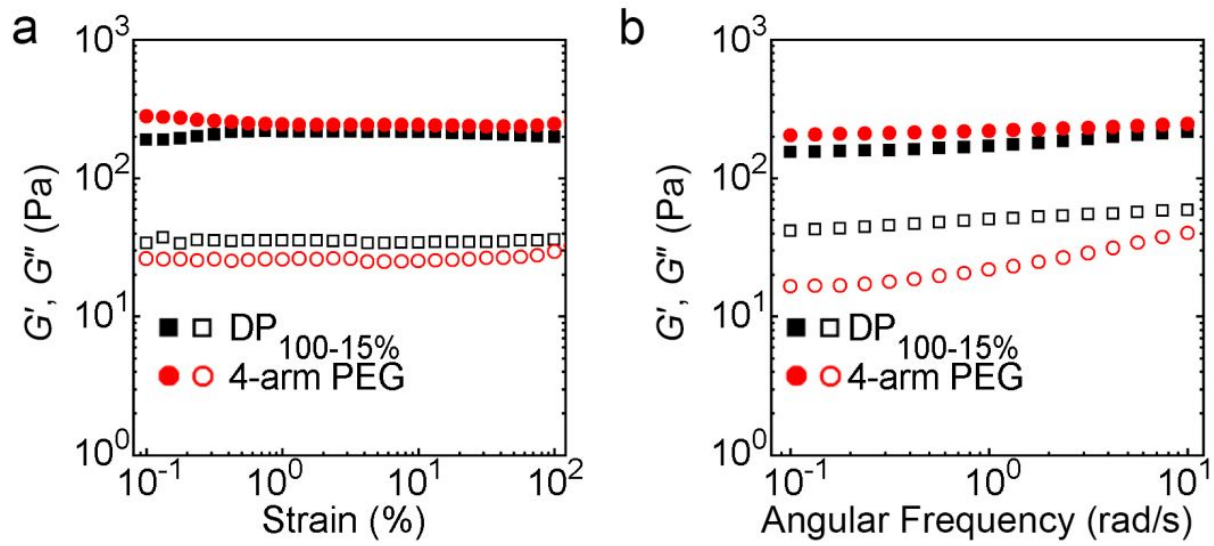

Figure S18. Strain dependent ( $\omega=10 \mathrm{rad} / \mathrm{s}, 25^{\circ} \mathrm{C}, 10 \mathrm{wt} \%$ ) (a) and frequency dependent $\left(\epsilon=5 \%, 25^{\circ} \mathrm{C}, 10 \mathrm{wt} . \%\right)$ rheology measurements (b) of $\mathrm{DP}_{100-15 \%}$ and 4-arm PEG hydrogels. $G^{\prime}$, solid dots; $G^{\prime \prime}$, empty dots. 


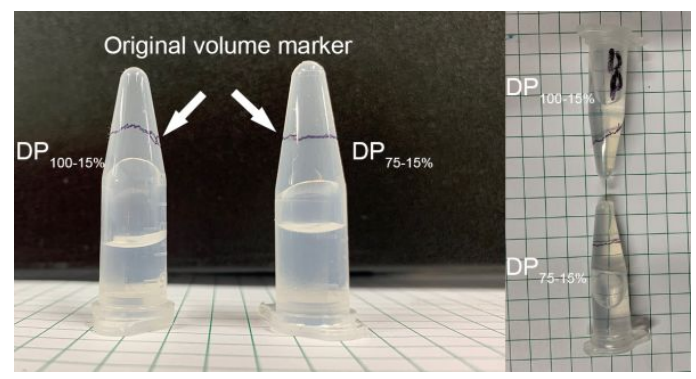

Figure S19. The measured $10 \mathrm{wt} \%$ brush polymer gels $\left(\mathrm{DP}_{75-15 \%}\right.$ and $\mathrm{DP}_{100-15 \%)}$ maintained their gel structures after months stored in PBS buffer. White arrows indicate the original volume before being immersed in the PBS buffer.
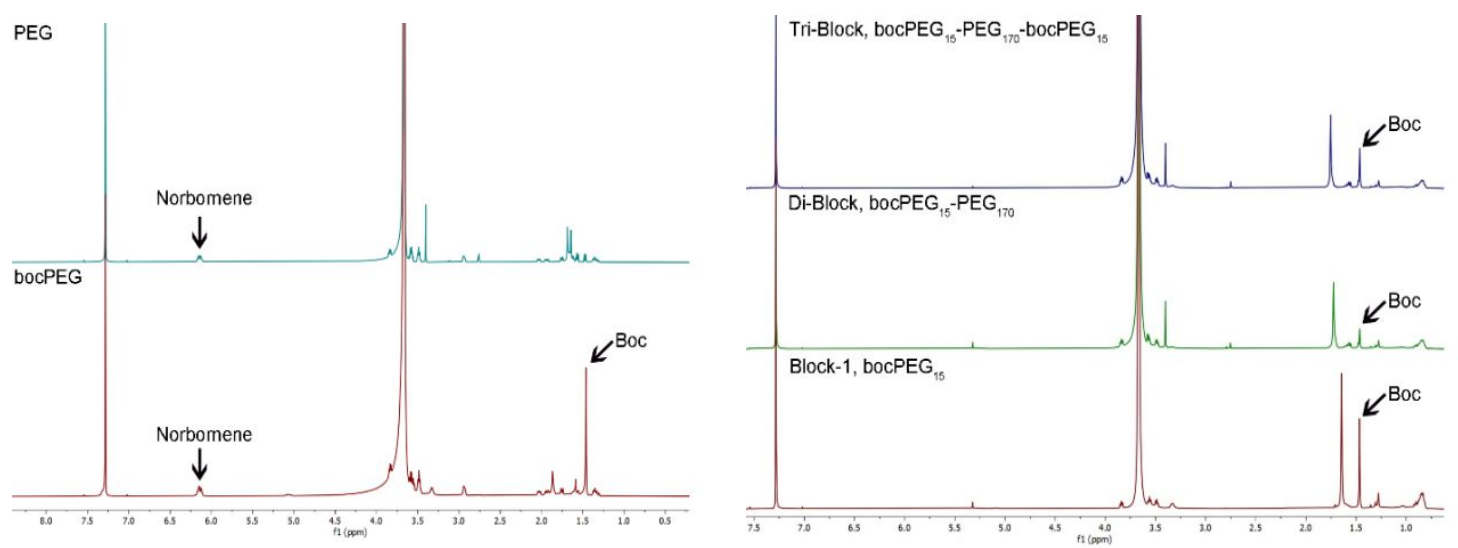

Figure S20. ${ }^{1} \mathrm{H}$ NMR spectrum of macromonomers (PEG and bocPEG) and each stage during triblock brush polymer synthesis (end-to-end connection fashion, 15\% BocPEG monomers).
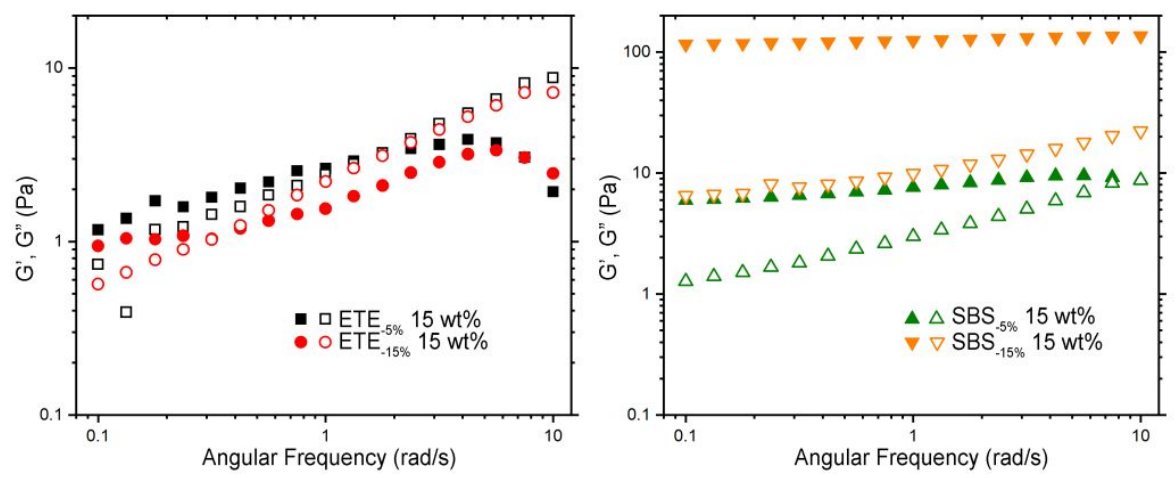

Figure S21. Frequency dependent $\left(\epsilon=5 \%, 25^{\circ} \mathrm{C}, 5\right.$ and $\left.15 \mathrm{wt} \%\right)$ rheology measurements of PEG hydrogels with different crosslinking schemes (end-to-end, left; and side-by-side, right). $G^{\prime}$, solid dots; $G^{\prime \prime}$, empty dots. 


\section{References:}

(1) Pontrello, J. K.; Allen, M. J.; Underbakke, E. S.; Kiessling, L. L. Solid-Phase Synthesis of Polymers Using the Ring-Opening Metathesis Polymerization. J. Am. Chem. Soc. 2005, $127,14536$.

(2) Lu, X.; Watts, E.; Jia, F.; Tan, X.; Zhang, K. Polycondensation of Polymer Brushes via DNA Hybridization. J. Am. Chem. Soc. 2014, 136, 10214-10217

(3) Love, J. A.; Morgan, J. P.; Trnka, T. M.; Grubbs, R. H. A Practical and Highly Active Ruthenium-Based Catalyst that Effects the Cross Metathesis of Acrylonitrile. Angew. Chem. Int. Ed. 2002, 41, 4035.

(4) Jha, S.; Dutta, S.; Bowden, N. B. Synthesis of Ultralarge Molecular Weight Bottlebrush Polymers Using Grubbs' Catalysts. Macromolecules 2004, 37, 4365-4374.

(5) Shibuya, Y.; Tatara, R.; Jiang, Y.; Shao-Horn, Y.; Johnson, J. A. Brush-First ROMP of Poly(ethylene Oxide) Macromonomers of Varied Length: Impact of Polymer Architecture on Thermal Behavior and $\mathrm{Li}^{+}$Conductivity. J. Polym. Sci. Pol. Chem. 2019, 57, 448-455. 